\title{
Discapacidad y calidad de vida en decisiones de triaje durante el COVID-19: marcos éticos de equidad y perspectivas queer-crip
}

Disability and quality of life in triage decisions during COVID-19: equity frameworks and queer-crip perspectives

Lautaro Leani

Universidad de Buenos Aires - Argentina

Buenos Aires, Argentina

lautaroleani@filo.uba.ar

\section{RESUMEN}

En estudios sobre economía de la salud es usual la utilización de las unidades de medida que ponderan la cantidad de años de vida esperados y la presencia de discapacidad (DALY) o la calidad de vida (QALY) durante dicho período. El objetivo de este trabajo es indagar en los supuestos y las consecuencias éticas del uso de estas unidades de medida como criterios de prioridad o desempate en decisiones de triaje durante la pandemia de COVID-19. Se abordará, en primer lugar, las conexiones entre estas categorías y la perspectiva estándar de la calidad de vida de las personas con discapacidad, según la cual la calidad de vida tales personas son comparativamente inferior a la del resto. En segundo lugar, se argumentará que dicha perspectiva es errónea y que, cuando influye en decisiones de triaje, retroalimenta injusticias estructurales no permisibles desde un marco ético de equidad. Por último, se discutirá la representación de la profundización de los mecanismos que producen desigualdad e injusticia estructural como un período de crisis que justifica estados de emergencia, excepción y urgencia. Esto permitirá mostrar que focalizar únicamente en la necesidad de aplicar criterios individuales de prioridad o desempate en decisiones de triaje durante el COVID-19 se presenta bajo una retórica engañosa que esconde los procesos sistemáticos de exclusión y explotación que han producido gran parte de las condiciones que generan los dilemas del presente, y que obtura medidas integrales, colectivas y a largo plazo.

Palabras clave: Discapacidad; Calidad de vida; Triaje; COVID-19; Ética

\section{ABSTRACT}

In health economics studies, it is usual to use measurement units that weight the number of years of life expected and the presence of disability (DALY) or the quality of life (QALY) during such a period. The objective of this work is to inquire into the assumptions and ethical consequences of the use of these units of measurement as criteria of priority or tiebreaker in triage decisions during the COVID-19 pandemic. First, it will address the connections between these categories and the standard view of the quality of life of people with disabilities, according to which the quality of life of these people is comparatively lower than that of the rest. Second, it will be argued that this perspective is wrong and that, when it influences triage decisions, it feeds back structural injustices that are not permissible from an ethical framework of equity. Finally, it will be discussed the representation of the deepening of the mechanisms that produce inequality and structural injustice as a period of crisis that justifies states of emergency, exception, and urgency. This will show that focusing solely on the need to apply individual priority or tiebreaker criteria in triage decisions during COVID-19 is presented under misleading rhetoric that hides the systematic processes of exclusion and exploitation that have produced a large part of the conditions that generate the dilemmas of the present, and that block comprehensive, collective and long-term measures.

Keywords: Disability; Quality of life; Triage; COVID-19; Ethics 


\section{Introducción}

Los Disability Adjusted Life Years (DALYs) y los Quality Adjusted Life Years (QALYs) son unidades de medida frecuentemente utilizadas en estudios sobre economía de la salud (Ortiz Amezquita y Plata Castillo, 2011; Thierer, 2019). Mientras que la primera de estas categorías pondera la cantidad de años de vida que se esperan de una persona y el grado de discapacidad que se presume que tendrá durante el tránsito de dichos años, la segunda pondera la cantidad de años de vida y la calidad de vida de tales años. En el primer caso, para determinar la unidad de valor "discapacidad" actualmente se utiliza una tabla de valores de discapacidad asignados a diferentes niveles de dificultad para realizar las actividades de la vida diaria, desarrollada originariamente por Murray en 1994, que ha sido actualizada a lo largo de distintas ediciones (Borruel, 2010; Mathers et al., 2006; Murray, 1994; World Health Organization, 2008). Para el caso de los QALYs, en cambio, la unidad de valor "calidad" se obtiene a partir de distintos mecanismos de encuesta poblacional. En Argentina, por ejemplo, se utiliza el instrumento EQ-5D-3L (Augustovski et al., 2017), desarrollado por el Grupo EuroQol, que pretende describir y valorar la "Calidad de Vida en Relación a la Salud" (Ministerio de Sanidad, Servicios Sociales e Igualdad de España, 2014).

Muchos artículos, guías y manuales consideran a las categorías de DALYs y QALYs como unidades de medida opuestas (Alvis y Valenzuela, 2010; Lee et al., 2017). Sostener que la presencia de discapacidad durante un período de tiempo supone una menor calidad de vida es altamente cuestionable, puesto que establece una correlación directa entre diversidad funcional y baja calidad de vida. Siguiendo a Moya Santander, utilizaré la expresión "personas con diversidad funcional", para referirme a aquellas "personas que con diferentes características biofísicas y dadas las condiciones de entorno generadas por la sociedad, deben realizar las mismas tareas o funciones que el resto de la sociedad de manera diferente" (Moya Santander, 2018). Este uso pretender ser operativo y no identitario, por lo que funciona como una campana semántica que engloba tanto a aquellas personas que se identifican con tal expresión, como a quienes no lo hacen pero que son comprendidas bajo su significado (Radi, 2019). Esta expresión general abarca a personas con múltiples condiciones tradicionalmente etiquetadas como "discapacidades", tales como "síndrome de Down, parálisis cerebral, acondroplasia, espina bífida, autismo, ceguera, sordera, paraplejia y cuadriplejia” (Campbell y Stramondo, 2017, p. 153; traducida por el autor).

En las últimas décadas, la forma en que los juicios sobre la calidad de vida de las personas con diversidad funcional acarrean múltiples prejuicios identitarios negativos ha sido largamente estudiada, tanto para exhibir las maneras en que estos juicios cierran las posibilidades simbólicas y materiales de futuro para dicho grupo social, como para denunciar un sistema de opresión estructural que los produce y es producido en estos, el capacitismo (Arnau Ripollés, 2014; Campbell, 2008; Fritsch, 2016; Kafer, 2013; Palacios y Romañach, 2008; Shakespeare, 2006; Stramondo, 2020; Wolbring, 2008). En este marco, el capacitismo es definido como:

...una red de creencias, procesos y prácticas que producen un tipo particular de "yo" y de cuerpo (el estándar corpóreo), que se proyecta como lo perfecto, lo típico de la especie y, por lo tanto, lo esencial y plenamente humano. La discapacidad, entonces, se presenta como un estado disminuido del ser humano (Campbell, 2001, p. 44).

Bajo este sistema social, las prácticas discursivas que evalúan la calidad de vida de las personas a partir de estereotipos identitarios se nutren de lo que Amundson llama la "perspectiva estándar de la calidad de vida de las personas con discapacidad" (standard view of QOL of disabled people), de aquí en adelante, perspectiva estándar de la discapacidad. Dicho punto de vista entiende, a partir de creencias falsas y criterios de dudosa legitimidad, que las características psicofísicas de las personas con diversidad funcional empeoran su calidad de vida (Amundson, 2005, 2010; Campbell y Stramondo, 2017; Coca Vila, 2021; Smith, 2020). 
Si bien es posible argumentar que en economía de la salud las categorías de DALYs y QALYs son relevantes para la planificación pública en materia de inversión en salud, "presentarían problemas éticos y legales" en muchos otros contextos, como sostienen Emanuel et al (2020, p. 2052). En este artículo, mi intención es evaluar uno en particular: la asignación de recursos escasos en decisiones de triaje. Mi hipótesis es que la aplicación de las categorías de DALYs y QALYs como criterios de prioridad o desempate en decisiones de triaje supone y refuerza injusticias estructurales que implican inequidades por desigualdad de consideración (unequal concern) y que lesionan la dignidad de las personas con diversidad funcional, puesto que privilegian un grupo social por sobre otro a partir de juicios acerca de la calidad de vida de un conjunto de personas sobre la base de presupuestos capacitistas propios de la perspectiva estándar de la discapacidad. Tal perspectiva carece de pertinencia y relevancia para determinar qué personas deben priorizarse frente a la disponibilidad escasa de recursos potencialmente salvadores, por lo que su utilización no es éticamente permisible y tampoco debe ser tenida en cuenta como criterio de desempate.

Respecto de la organización del artículo, se comenzará por mostrar las formas en las cuales las categorías de DALYS y QALYs constituyen gran parte de sus cálculos a partir de la perspectiva estándar de la discapacidad, según la cual la calidad de vida de las personas con diversidad funcional es inferior a la del resto. En segundo lugar, se argumentará que dicha perspectiva es falaz y que, cuando influye en decisiones de triaje, retroalimenta injusticias estructurales no permisibles desde un marco ético de la equidad, puesto que lesionan la dignidad y los consecuentes derechos humanos. Por último, se discutirá la representación de la profundización de los mecanismos que producen desigualdad e injusticia estructural como un período de crisis que justifica estados de emergencia, excepción y urgencia. Esto permitirá mostrar que focalizar únicamente en la necesidad de aplicar criterios individuales de prioridad o desempate en decisiones de triaje durante el COVID-19 se presenta bajo una retórica engañosa que esconde los procesos sistemáticos de exclusión y explotación que han producido gran parte de las condiciones que generan los dilemas del presente, y que obtura medidas integrales, colectivas y a largo plazo.

\section{Metodología}

La metodología de este trabajo es propia de los artículos teóricos que hacen uso de investigaciones y desarrollos teóricos previos para aportar nuevos modos de comprender un fenómeno complejo. De esta forma, tanto los datos como las teorías fueron seleccionados con el objetivo de examinar los presupuestos implícitos y las consecuencias éticas del uso de los DALYs y QALYs en decisiones de triaje durante la pandemia de COVID-19. La selección de las fuentes prioriza a aquellas producidas desde organismos internacionales, organizaciones activistas de derechos, organismos científicos nacionales y revistas científicas certificadas a partir de criterios metodológicos de veracidad, como la cantidad de personas abarcadas en los estudios, la solidez metodológica y la actualidad de los datos relacionados con la temática. Bajo este marco metodológico, es posible identificar dos tipos de fuentes: por un lado, aquellas que sirven como objeto de análisis para dar cuenta del fenómeno en cuestión, entre las que encontramos a las guías, los datos estadísticos, los estudios sobre economía de la salud y los informes periodísticos. Por otro lado, aquellas fuentes que brindan las herramientas teórico-metodológicas para el análisis de las fuentes objeto, como las tradiciones teóricas, los documentos de organismos internacionales y los conceptos filosóficos. Así, el desarrollo de este trabajo sigue una metodología que funde estos dos tipos de fuentes para abordar las características fundamentales del tema abordado, examinar ensayos que lo anteceden, aportar interrogantes para la discusión en nuevos términos y, finalmente, ensayar posibles respuestas. 


\section{Desarrollo}

\subsection{Calidad de vida y perspectiva estándar de la discapacidad}

En el contexto particular de las decisiones de triaje durante la pandemia de COVID-19, algunas instituciones y comités han incluido entre los criterios de prioridad o desempate a las categorías de DALY y QALY. La Sociedad Española de Medicina Intensiva, Crítica y Unidades Coronarias (SEMICYUC) recomienda que "ante dos pacientes similares, se debe priorizar a la persona con más años de vida ajustados a la calidad (AVAC) o QALY (Quality-Adjusted Life Year)" (SEMICYUC, 2020, p. 11). Otros profesionales afirman que los QALYs y DALYs "dan una idea de las metas generales, que se deben tener siempre en perspectiva, cuando se está en circunstancias de priorizar pacientes que obtendrán mayor beneficio del cuidado médico en [Unidad de Cuidados Intensivos]" (Burdiles Pinto y Ortiz Pommier, 2021, p. 70). Por último, como evidencia la asociación Disability Rights Washington, varias guías distribuidas por el Departamento de Salud del Estado de Washington (WA DOH) sostienen que, ante la disponibilidad escasa de recursos potencialmente salvadores, es recomendable derivar a pacientes hospitalarios con "pérdida de reservas de energía, capacidad física, cognición y salud general” a cuidados ambulatorios o paliativos, en lugar de brindarles parte de los recursos potencialmente salvadores de escasa disponibilidad (Disability Rights Washington, 2020, p. 2).

Si bien las guías que recomiendan la utilización de tales categorías no presentan una fundamentación explícita de las razones de su uso en decisiones de triaje para la pandemia de COVID-19 (White y Lo, 2020), la forma en que los DALYs y QALYs son calculados permite explicar, en parte, su incorporación como criterio. El valor asignado al nivel de discapacidad en los DALYs es medido a partir del grado de asistencia o apoyo requerido para realizar diversas actividades cotidianas, por lo que si los DALYs son entendidos como "un mal que debe ser minimizado" (Burdiles Pinto y Ortiz Pommier, 2021, p. 70), se puede deducir que la realización de actividades con asistencia forma parte de ese mal a disminuir, incluso cuando ello implique, en casos de escasez de recursos, excluir a las personas con mayor índice de DALYs del acceso a estos. Asimismo, el "mal que debe ser minimizado" en el caso de los QALYs es obtenido a partir de encuestas que realizan preguntas en torno a parámetros de independencia, eficacia y productividad, que si bien pretenden incorporar la opinión subjetiva de cada persona encuestada, luego se interpreta como "la opinión pública general”, se establece como una valoración fiable y democrática, y se utiliza como criterio, muchas veces sin mediar un análisis crítico. Gran parte de los fundamentos de estas asignaciones valorativas y sus instrumentalizaciones son rastreables a partir del desglose de la perspectiva estándar de la discapacidad. Según Wieseler, dicha perspectiva es una creencia común, altamente respaldada por filósofos/as, intelectuales y bioeticistas, que considera que la discapacidad tiene un impacto negativo en el bienestar de las personas y que sería recomendable eliminarla de la experiencia humana (Wieseler, 2020). Además,

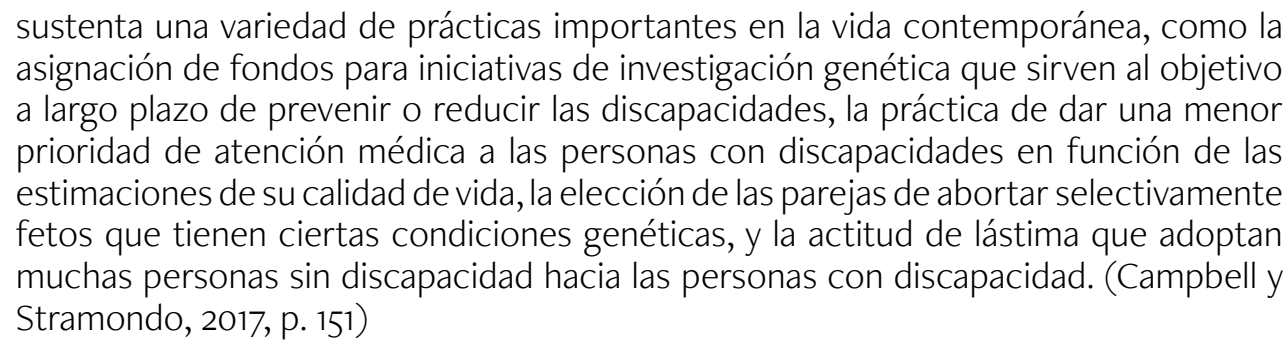

La influencia de esta perspectiva determina, en gran parte, la forma en que las personas con diversidad funcional serán percibidas, lo que también repercute en cómo ellas mismas se autopercibirán. Como sostiene Fanon en relación al racismo, la propia imagen se constituye a partir de "la mirada del otro" y el conocimiento del propio cuerpo es una actividad negadora en la que "el 
conocimiento es en tercera persona" (Fanon, 2009, p. 112). Es así como las relaciones establecidas por las mediciones de los DALYs y QALYs tienen un efecto performativo tanto en las personas que ven su calidad de vida comprendida como inferior, como en aquellas que se benefician de estas escalas. Para Butler, el aspecto performativo de los enunciados lingüísticos cita normas que, al ser cumplidas exitosamente o no por las personas, se siguen reiterando y adquieren cada vez mayor legitimidad y naturalización. De esta forma, los enunciados lingüísticos y las prácticas asociadas a una identidad específica se conforman a partir de la repetición constante de las normas, logrando que lo que éstas dicen sean las únicas formas de pensar e imaginar dicha identidad (Butler, 1998, 2007). En este sentido, cuando se reflexiona en torno a la producción social de la diversidad funcional, lo que se afirma es que ésta "no existe como realidad por fuera de los dispositivos que la construyen como categoría subjetivante y de asignación de identidad" (Yarza de los Ríos et al., 2019, p. 23).

En el caso particular de las categorías de DALYs y QALYs, el refuerzo de los estereotipos identitarios negativos sobre las personas con diversidad funcional se da a partir de asignaciones de valor. $\mathrm{Si}$ en las tablas informativas que miden el valor "discapacidad", la "necesidad de asistencia en actividades cotidianas como comer, higiene personal y vestido" tiene un puntaje de 0,92 y la categoría inmediatamente siguiente es la "muerte" (Borruel, 2010; Murray, 1994), el peso negativo de la diversidad funcional se presenta como evidente. Estas valoraciones que se presumen como meramente descriptivas o asertóricas funcionan, retomando a Butler, de forma performativa, por lo que generan la naturalización de aquellos prejuicios identitarios ya previamente formados en un sistema social regido por estereotipos capacitistas. En otras palabras, que la necesidad de asistencia sea entendida como el peor escenario posible sólo detrás de la muerte refuerza los preconceptos capacitistas de la persona que lo lee, puesto que ahora entiende que su postura personal, influida por estereotipos identitarios negativos, está científicamente legitimada. En el caso de instituciones epistémicamente autorizadas, como la medicina, la economía de la salud o la bioética, el éxito del efecto performativo está garantizado desde el inicio, puesto que cuentan con la legitimidad suficiente como para citar normas previas que reiteran la perspectiva estándar de la discapacidad a título de "expertas".

De esta forma, lo descriptivo manifiesta su aspecto performativo, y produce la creencia que identifica una vida saludable y de calidad con una vida "sin discapacidad". Así, aspirar a vivir "sin discapacidad" se muestra como un imperativo social que se cristaliza en lo que McRuer llama "capacidad corporal obligatoria", un ideal regulativo que entiende al cuerpo como una "máquina", con partes que funcionan de cierta forma, y que se presenta como el cuerpo "natural" y, por añadidura, el cuerpo “normal” (McRuer, 2020). Desde un análisis histórico, la valoración de aquellos cuerpos que se ajustan a los criterios de utilidad, productividad y aptitud laboral surge a partir de la división social del trabajo capitalista desarrollada desde Europa a partir del siglo XVIII. Dado el carácter central que la producción de bienes y servicios poseía, y aún posee, en tales sociedades, la diversidad funcional ha sido significada como una "tragedia personal" que legitima simbólicamente una presunta inferioridad corporal y que instaura "la normalidad como modo de reconquista de la humanidad perdida” (Yarza de los Ríos, et al., 2019, p. 35). Es así como la introducción de la normalidad en el sistema social inserta la capacidad corporal obligatoria como imperativo social, en tanto que ser una persona leída como "normal" implica la posibilidad de concreción del resto de las aspiraciones sociales, como el acceso a la educación, a la salud, a un trabajo remunerado, a ser sujeto y objeto de deseo, a ser reconocido como sujeto epistémico, etc. En otras palabras, "la obligatoriedad es producida y ocultada con la apariencia de la elección, mistificando un sistema en el que, en realidad, no hay opción” (McRuer, 2006, p. 7).

Es por ello que la medición de la salud de una persona en relación a su "incapa[cidad] de realizar [las] actividades de todos los días", como figura en la encuesta que determina el valor de la "Calidad de Vida en Relación a la Salud", en los QALYs (Sociedad Española de Reumatología, 
2015), correlaciona las nociones de "vida saludable" y de "vida de calidad" con la de "vida independiente". Esto renueva la demanda social de una respuesta afirmativa a la pregunta que la cultura capacitista le repite constantemente a las personas con diversidad funcional: "Sí, pero a fin de cuenta, ¿no preferirías ser más como yo?” (McRuer, 2006, p. 9). De esta forma, desear la diversidad funcional se vuelve un absurdo en una cultura que "promueve prácticas, metáforas y narrativas encaminadas a vivirla como sufrimiento y padecimiento" a partir de mandatos sociales que fabrican "la falsa idea de que los cuerpos sin discapacidad son de alguna manera perfectos, sanos y radicalmente autosuficientes o, quizá, precisamente, más válidos y, por tanto, más importantes para la sociedad" (Maldonado Ramírez, 2020, p. 53).

\subsection{Injusticia estructural, inequidad y dignidad}

Una vez trazada la conexión entre las categorías de DALYs y QALYs y la perspectiva estándar de la discapacidad, se pasará a describir la forma en que este punto de vista sostiene creencias falsas que, cuando son instrumentalizadas como criterios en decisiones de triaje, retroalimentan injusticias estructurales no permisibles desde un marco ético de la equidad, puesto que lesionan la dignidad y los consecuentes derechos humanos de las personas con diversidad funcional.

Como sostiene Wieseler, la perspectiva estándar iguala discapacidad con sufrimiento, dolor y/o enfermedad. Sin embargo, estas relaciones son falsas. En primer lugar, la evidencia empírica demuestra que las personas con diversidad funcional han calificado su propia calidad de vida de manera similar o apenas por debajo del promedio de la población en general (Wasserman et al., 2016; Wieseler, 2020). Para Amundson, esta negativa, por parte de múltiples bioeticistas, a reconocer la calidad de vida que manifiestan las personas con diversidad funcional, incluso cuando cuentan con un entorno no discapacitante, se explica por prejuicios modelados por estigma social e ignorancia, más que por datos empíricos (Amundson, 2005; Naciones Unidas, 2019).

En segundo lugar, la asociación de la discapacidad con el dolor y la enfermedad es contingente y depende exclusivamente de cada condición específica. Existen muchos tipos de discapacidad que no implican dolor ni presencia de ninguna enfermedad asociada (Campbell y Stramondo, 2017; Wasserman et al., 2016). Incluso en gran parte de los casos en los que ello ocurre, los tratamientos médicos en combinación con los entornos adecuados permiten un tránsito aceptable. Además, la exigencia de la perspectiva estándar de la discapacidad de vivir sin ningún tipo de dolor no es una aspiración alcanzable por ninguna persona, incluso cuando ella no transite por ninguna intervención o enfermedad dolorosa en toda su vida. Por último, quienes sostienen que la diversidad funcional es causa de sufrimiento porque impide el goce de ciertos bienes, como "apreciar un cuadro" en el caso de la ceguera, no tienen en cuenta que dichas personas pueden seguir disfrutando de otros múltiples bienes que hacen sus vidas igualmente deseables, como “apreciar una melodía” (Stramondo y Campbell, 2020). Las formas en que optamos entre qué bienes deseamos adquirir, y cuáles no, están influenciadas por múltiples factores, y acusar a la diversidad funcional como causante de sufrimiento por impedir algunos de ellos es sumamente tendencioso.

Por su parte, White y Lo identifican que algunas recomendaciones estatales y guías de profesionales sugieren excluir, sin una justificación adicional a la necesidad de establecer criterios de prioridad, a ciertos pacientes con determinadas condiciones de salud, como enfermedad renal en etapa terminal o deterioro cognitivo severo, a partir de la aplicación selectiva de criterios relacionados con su pronóstico a largo plazo o su funcionalidad (White y Lo, 2020). Si se comprende que las categorías de DALYs y QALYs no son suficientes para justificar la exclusión de ciertos tipos de pacientes de la asignación de recursos potencialmente salvadores, su utilización constituye una práctica que retroalimenta injusticias estructurales que afectan a dichos grupos sociales. 
Según sostiene Holzer, a diferencia de la injusticia transaccional, la injusticia estructural se caracteriza por una explotación sistemática de un grupo social por sobre otro, que engloba factores estructurales como las barreras de acceso a la educación, la salud y la participación política o las inequidades distributivas. Para comprender mejor este fenómeno, la autora propone un patrón de probabilidad condicional que establece que, a factores y variables iguales, cuando la presencia de explotación transaccional se incrementa significativamente bajo condiciones injustas entre participantes, respecto a su presencia contrafáctica en condiciones justas, existe una injusticia estructural. La explotación transaccional mutuamente ventajosa ocurre cuando en un intercambio o transacción entre dos agentes, el agente explotador resulta inequitativamente beneficiado en detrimento del agente explotado. Bajo esta concepción, hay injusticia estructural cuando la probabilidad de que ocurra una injusticia transaccional bajo condiciones de trasfondo injustas entre participantes es significativamente mayor a la probabilidad de que ocurra bajo condiciones de trasfondo justas. Si, por el contrario, la probabilidad de ocurrencia de los casos de explotación transaccional bajo contextos injustos es similar en contextos justos, estamos ante injusticias transaccionales no sistemáticas y, por lo tanto, no se puede afirmar la presencia de injusticia estructural (Holzer, 2020).

Las injusticias estructurales han sido largamente estudiadas bajo interpretaciones marxistas en las que la injusticia ocurre en la distribución de recursos. Sin embargo, autoras como Iris Young han extendido dicha categoría para mostrar desigualdades estructurales en torno a otras cuestiones como el género y la raza (Holzer, 2020). Retomando la conceptualización de Holzer, las injusticias estructurales se producen a partir de prácticas sistemáticas que aumentan la probabilidad, para ciertos sectores identitariamente marcados, de ser objeto de injusticia transaccional. En este sentido, la injusticia estructural ejercida sobre las personas con diversidad funcional, retroalimentada por la perspectiva estándar de la discapacidad, garantiza una explotación omnipresente en sus interacciones sociales, como lo muestran diversos informes (Naciones Unidas, 2019). Así, este grupo social debe afrontar sistemáticamente una suma inequitativa de riesgos, cargas y costos evitables para acceder a los mismos derechos básicos que el resto de la población.

Según establece la Organización Panamericana de la Salud (OPS), garantizar la equidad en la salud debe ser entendido como un objetivo integral que toma en cuenta la forma en que diversos ámbitos sociales influyen en la salud de grupos sociales determinados (OPS, 2020). Como escriben sus autores, "la equidad en la salud es la ausencia de diferencias evitables o remediables entre grupos de personas definidos desde un punto de vista social, económico, demográfico o geográfico" (OPS, 2020, p. 9). Estas diferencias tienen como principal origen a las injusticias estructurales, que perpetúan desigualdades sistemáticas, tanto en la distribución de recursos, como en el acceso, permanencia y participación en las distintas instituciones que conforman una sociedad. A partir de la distinción clásica establecida por Dworkin, entre igual consideración (equal concern) e igual respeto (equal respect), se puede comprender en qué sentido la utilización de los DALYs y QALYs en decisiones de triaje reproducen injusticias estructurales no permisibles desde un marco de equidad. Para Dworkin, existen dos principios de dignidad que conforman la moralidad política: el autorrespeto y la responsabilidad. De dichos principios se derivan las obligaciones ético-políticas del Estado, y otros organismos soberanos, de tratar a las personas que caen bajo su gobierno con igual grado de importancia y preocupación por el destino de cada una, y con igual grado de respeto por el derecho de cada persona de "hacer algo de valor" con su vida (Dworkin, 2010). Es por ello que bajo el marco de la equidad dworkiniano, la dignidad se erige como el fundamento último que explica la obligación ética de una sociedad equitativa.

La forma en que la dignidad y los derechos humanos se entrelazan para justificar la obligación ética de la equidad en varias de las convenciones, declaraciones e informes de organizaciones es entendida por Gilabert desde dos interpretaciones de la dignidad: como dignidad-estatus 
(status-dignity) y como dignidad-condición (condition-dignity). El primero es un valor intrínseco que fundamenta cierto tipo de trato hacia quien lo posee, equivalente al autorrespeto en Dworkin, mientras que el segundo, es un estado de cosas bajo el cual podemos afirmar que sus derechos humanos, derivados de su dignidad-estatus, están cumplidos. Tomando el marco ético de las capacidades desarrollado por Nussbaum, el autor sostiene que las personas poseen algunas de las capacidades generales que justifican su dignidad-estatus, como la evaluación, reconocimiento y actuación basadas en razones, la toma de decisiones en torno a sus planes de vida, la generación de formas alternativas de vida personal y social, la experimentación de placer y afecto, el establecimiento de relaciones cognitivas y estéticas con el mundo que las rodea, entre muchas otras. En este contexto, los derechos humanos se erigen como una obligación ética que garantiza el resguardo necesario para que dichas capacidades puedan ejercerse (Gilabert, 2015b). En el esquema argumentativo que propone Gilabert,

1. Las personas humanas tienen dignidad-estatus en virtud de algunas de sus características más o menos generales, tanto constantes como permanentes.

2. Las personas humanas tienen derechos humanos porque tienen dignidad-estatus. 3. Los derechos humanos son aquellos derechos cuyo cumplimiento asegura que las personas humanas disfruten de una vida digna (dignidad-condición).

4. Las personas humanas tienen el deber de respetar, proteger y promover el cumplimiento de esos derechos. Tales deberes pueden tener un alcance y un sitio amplios.

5. Para asegurar el cumplimiento de los derechos humanos, las personas humanas requieren acceso a diversas formas de poder y, por lo tanto, tienen derecho a ese acceso (Gilabert, 2015b, pp. 201-202).

En la misma línea, y en oposición a la valoración negativa del requerimiento de apoyo que encontramos en el cálculo de los DALYs y QALYs, Butler entiende que la vulnerabilidad, como circunstancia asociada a la interdependencia y la precariedad, no es un estado pasajero o alterable, sino una condición que coexiste con nuestra existencia misma, ya que nombra una manera de relacionarse con el mundo. Reconocernos vulnerables implica aceptar "nuestra dependencia fundamental no sólo de otros, [sino] también de un mundo que nos sostiene y que es sostenible" (Butler, 2017, p. 23). Así, la apertura a recibir y brindar asistencia o apoyo forma parte de las capacidades humanas que constituyen nuestra dignidad-estatus, por lo que no necesitamos ganarnos la consideración y el respeto a partir del cumplimiento de exigencias sociales como la independencia y la productividad en nuestras actividades (Extremo Ruano, 2016). La perspectiva estándar de la discapacidad tuerce la idea de dignidad, en tanto obtener apoyo se entiende como una fuente de humillación, vergüenza o baja calidad de vida, y brindarlo, como una fuente de arrogancia o culpa. Este punto de vista tiende a retroalimentar las relaciones existentes de inequidad, de injusticia estructural y de indiferencia (Gilabert, 2015a).

\subsection{Figuración de crisis e inequidades preexistentes}

Incluso desde aquellos sectores que comparten la postura de que la utilización de las categorías de DALYS y QALYs en decisiones de triaje no es éticamente permisible, sostienen que la urgencia de la "crisis del COVID-19" provoca la necesidad de establecer múltiples criterios de maximización de vidas salvadas sobre personas que, ante tal escenario, son igualmente valiosas y prioritarias. En este sentido, son variados los informes, guías, artículos y portales de noticias que presentan a la pandemia de COVID-19 como una etapa de crisis (Redacción LAVOZ, 2021; Rivera López et al., 2020; Savin y Guidry-Grimes, 2020; Saxena et al., 2021; Scientific Advisory Group for Emergencies, 2020; SEMICYUC, 2020; Smith-Spark y Gan, 2021; VOX Media, 2021; WA DOH, 2020; Wilkinson, 2020). Bajo esta retórica, el período atravesado por la pandemia es entendido como un escenario que exige medidas drásticas e inmediatas para paliar sus efectos dañinos. La necesidad de establecer criterios en decisiones de triaje se muestra como una exigencia práctica que puede 
llegar a justificar, bajo la idea de "crisis", la utilización de categorías o valoraciones aparentemente despolitizadas que mantienen injusticias estructurales y que producen inequidades que afectan específicamente a ciertos grupos sociales que ya venían padeciendo procesos sistemáticos de violencia y exclusión. Si se tiene en cuenta que las inequidades en materia de salud que señala la OPS (2020) preceden a la situación actualmente presentada como “crisis", la desarticulación de esta categoría se muestra como particularmente pertinente.

Para comprender el funcionamiento de este mecanismo discursivo se entenderá que la figuración de la pandemia de COVID-19 como un "período de crisis" supone tres aspectos interrelacionados: la emergencia, la excepción y la urgencia. El primero se caracteriza por presentar a la crisis como una novedad, lo que tiende a desconectar la situación presente de las injusticias estructurales del pasado que produjeron su ocurrencia paulatina. La excepción exhibe a la crisis como un caso sin precedentes, lo que permite justificar medidas extraordinarias presentadas como despolitizadas y pasajeras. Finalmente, la urgencia representa al período de crisis como un fenómeno que requiere medidas inmediatas y que exige, bajo esta interpretación reduccionista, relegar las medidas estructurales, colectivas y a largo plazo a una "sala de espera". Así,

\begin{abstract}
...presentar una problemática social como algo pasajero que puede ser recortado en el tiempo, por un lado, desconoce el arraigo de dicha problemática en el tejido social y la historia, su funcionalidad de larga data, y por el otro justifica intervenciones "de urgencia" que abren exclusas (disciplinamiento, represión, control, coerción, eugenesia pasiva) que luego no se cerrarán (Pérez, 2020).
\end{abstract}

La identificación de estas continuidades entre injusticias estructurales constantes y períodos presentados como "crisis" permite exponer aquellos factores ocultos por esta retórica. En este sentido, las inequidades que afectan de manera desigual a distintos grupos sociales en la distribución económica y la participación política pueden ser exploradas a partir de la categoría de "muerte atmosférica". Según Fanon, quien padece una muerte atmosférica percibe la vida no como un florecimiento y desarrollo personal, sino como "una lucha permanente contra una muerte omnipresente y constante que es materializada en la hambruna generalizada, el desempleo, un nivel alto de muerte en su comunidad, el complejo de inferioridad y la ausencia de esperanza para el futuro" (Fanon, 2001, p. 115). En esta misma línea, Berlant sostiene que la muerte lenta de los sectores sociales más vulnerados ocurre en el ambiente atemporal de la vida en curso, donde las desigualdades estructurales están dispersas y cuyo desarrollo implica un presente ordinario de repetición y desgaste en el cual no hay eventos específicos que determinen una temporalidad discreta. En dicho ambiente, la dialéctica entre la agencia y la estructura se manifiesta en repeticiones predecibles y prácticas espaciales conformadas por episodios que, por separado, no cambian demasiado nada (Berlant, 2007). A diferencia de la conceptualización clásica de la muerte en contraste con la vida, y de la crisis en contraste con el desarrollo, las nociones de "muerte lenta" y de "muerte atmosférica" plantean que la muerte y la reproducción de la vida ordinaria son coextensivas, y que los períodos presentados como "crisis" esconden sus profundas conexiones con las figuraciones simbólicas del “desarrollo".

A partir de esta caracterización, es posible conectar la llamada "crisis del COVID-19" con aquellas condiciones que afectan de manera desigual a ciertos grupos sociales y que han generado, al menos en parte, los dilemas bioéticos del presente, como el desfinanciamiento del sistema de salud público, la precarización laboral y el incremento del trabajo informal, la suba de impuestos y del transporte, la exclusión del acceso, permanencia y participación en las instituciones sociales y los espacios de decisión, la monopolización del mercado de salud privado, entre muchas otras (OPS, 2020). La “emergencia" de la "crisis", entonces, se presenta como tal cuando la violencia y exclusión superan el umbral de lo socialmente permitido, es decir, cuando afectan a aquellos 
individuos que "no debería". Sin embargo, antes y después de la "crisis", un enorme porcentaje de la población continúa expuesto a los ambientes atemporales de la muerte lenta (Pérez, 2020).

Así, cuando este tipo de violencia discreta (Valverde Gefaell, 2015), que es ejercida a partir de procesos de muerte lenta que mantienen a grupos enormes de la población en condiciones de flexibilización y hasta exclusión de sus derechos humanos básicos, alcanza a sujetos socialmente mejor posicionados, la situación es presentada como un estado de emergencia que requiere de medidas excepcionales, generalmente avocadas a "recuperar la economía" o a "combatir un enemigo común" (AIDS Healthcare Foundation, 2021; Naciones Unidas, 2021), lo que pretende igualar los actores sociales y, en consecuencia, hacerlos igualmente responsables. Para Valim, los estados de excepción permiten justificar políticas anti-populares que subordinan la política a la economía (Valim, 2018), por lo que no es casual que, por ejemplo, las grandes industrias farmacéuticas, en complicidad con los estados nacionales, se nieguen a liberar las patentes de los tratamientos y vacunas contra el COVID-19, a pesar de las 3.7 millones de personas ya fallecidas (Médicos Sin Fronteras, 2021). Las inequidades geopolíticas actuales en el reparto de los recursos médicos son una muestra más de las continuas inequidades socio-económicas que las preceden.

En este sentido, sostener desde una retórica de crisis, que todas las vidas requieren de igual prioridad, pretendiendo que las decisiones en salud no estén influidas por problemas de injusticia estructural e inequidad, puede llegar a ser una trampa. Estos discursos, que intentan igualar a todas las personas para "combatir un enemigo común", en realidad podrían estar obturando medidas integrales, colectivas y a largo plazo que permitan remediar o evitar el mantenimiento de las inequidades preexistentes. Según los “Objetivos de Desarrollo Sustentable” de la Organización de las Naciones Unidas, los sectores de la población más damnificados durante la pandemia de COVID-19 continúan siendo los mismos que en períodos anteriores: las personas mayores, las personas con discapacidad, los/as niños/as, las mujeres y las personas migrantes y refugiadas (Naciones Unidas, 2020). Es por ello que destinar mayores recursos en sectores que histórica y estructuralmente han sido vulnerados no implicaría oponerse a establecer un modelo de triaje, sino que sería una decisión ético-política complementaria. Aunque ésta no maximice la cantidad de vidas salvadas en lo inmediato, como recomienda la mayoría de las guías aquí abordadas, podría erigirse como un compromiso con los valores éticos de justicia y equidad en el nivel apropiado, es decir, estructural, en lugar de priorizar únicamente el nivel individual, como lo sugiere la retórica de la crisis. Las medidas comprometidas con la esfera estructural apuntan a conseguir, en el mediano y largo plazo, una maximización de vidas salvadas que alcance a un mayor número de sujetos en una mayor complejidad de sus relaciones sociales, en comparación con políticas de "salvación" de sujetos individuales sometidos a triaje, que son necesarias, pero no suficientes para el cumplimiento de estos objetivos.

\section{Conclusiones}

Como se muestra en este trabajo, el uso de las categorías de DALYs y QALYs como criterios para dirimir en torno a pacientes con COVID-19 suele presentarse como una respuesta adecuada a la urgencia de la crisis sanitaria. Sin embargo, bajo el análisis expuesto, estas categorías, por un lado, están implícitamente constituidas por prejuicios identitarios negativos comprendidos bajo la perspectiva estándar de la discapacidad. Por otro lado, son usadas como criterios en decisiones de triaje sin ser sometidas a un análisis de las condiciones de inequidad estructural que las preceden. Teniendo en cuenta la influencia de la perspectiva estándar de la discapacidad en una gran variedad de prácticas importantes de la vida contemporánea, se comprende que produce efectos performativos tanto en las personas que son negativamente categorizadas, como en aquellas con las cuales interaccionan socialmente. A través de la naturalización y normalización de los estereotipos, esto que genera un imperativo social llamado "capacidad corporal obligatoria". Además, la perspectiva estándar de la discapacidad es falaz por al menos tres razones: carece de 
evidencia empírica, sus conexiones con el dolor y la enfermedad son contingentes y, en muchos casos, evitables o remediables, y su exigencia de disponibilidad del goce de todos los bienes es sumamente demandante y tendenciosa.

La aplicación de los DALYs y QALYs como criterios en decisiones de triaje no es éticamente permisible. Esto se evidencia a partir del concepto de injusticia estructural, entendida como una explotación sistemática sobre un grupo social determinado. Cuando la perspectiva estándar de la discapacidad sirve como justificación oculta para excluir del acceso a recursos sanitarios a las personas con diversidad funcional, se retroalimentan injusticias estructurales que reiteran y sistematizan instancias de explotación dirigidas hacia dicho grupo social. Por su parte, el marco de la equidad permite identificar que dichas injusticias son remediables o evitables y que, cuando se producen, incumplen el deber ético de respetar, proteger y promover los derechos humanos que se basan en la dignidad-estatus de las personas. Por último, un examen de la retórica de la pandemia de COVID-19 figurada como "crisis" logra desocultar aquellas injusticias estructurales que producen inequidad y que son condición necesaria de los dilemas bioéticos del presente. A partir de este discurso, se legitiman medidas de emergencia, excepción y urgencia que simulan la igualación de todas las personas con el fin de "combatir un enemigo común", pero que en realidad obturan formas alternativas de pensar en políticas integrales, colectivas y a largo plazo.

Es por ello que estimo que, para desarrollar medidas que superen la inmediatez de estos planteos recurrentes, será fundamental tener en cuenta la capacidad performativa y normativa de las decisiones en bioética, y su impacto en las estructuras sociales que producen desigualdades injustas. Además, las personas perjudicadas podrán reivindicar sus derechos y reforzar las resistencias colectivas que ya vienen oponiendo a estas inequidades únicamente en tanto dejen de ser excluidas de los espacios de participación política y de decisión institucional. Solo teniendo en cuenta estas conexiones inescindibles, considero posible hacer frente al desafío del trabajo simultáneo en el que las respuestas a los problemas urgentes no impidan una transformación social profunda.

\section{REFERENCIAS}

AIDS Healthcare Foundation. (2021). Es hora de declararle la guerra al COVID-19. Business Wire, Comunicados de Prensa. https://bwnews.pr/3eNpHS2

Alvis, N. y Valenzuela, M. T. (2010). Los QALYs y DALYs como indicadores sintéticos de salud. Revista Médica de Chile, 138(2), 83-87. https://doi.org/10.4067/So034-98872010001000005

Amundson, R. (2005). Disability, ideology, and quality of life. En D. Wasserman, J. Bickenbach, y R. Wachbroit (Eds.) Quality of life and human difference: Genetic testing, health care, and disability. (pp. 101124). Cambridge University Press.

Amundson, R. (2010). Quality of Life, Disability, and Hedonic Psychology. Journal for the Theory of Social Behaviour, 40(4), 374-392. https://doi.org/10.1111/j.1468-5914.2010.00437.x

Arnau Ripollés, S. (2014). iDis-capacidad “a la carta”! Wannabes. ¿Una cuestión Bioética? En F. J. León Correa, D. León Rábago, y V. E. Navarrete Cruz (Coords.) Bioética para la toma de decisiones Parte I. (pp. 457-483). Federación Latinoamericana de Instituciones de Bioética (FELAIBE).

Augustovski, F., Colantonio, L. D., Galante, J., Bardach, A., Caporale, J. E., Zárate, V., Chuang, L. H., Pichon Riviere, A., y Kind, P. (2017). Measuring the Benefits of Healthcare: DALYs and QALYS - Does the Choice of Measure Matter? A Case Study of Two Preventive Interventions. International Journal of Health Policy and Management, 6(x), 1-17. https://doi.org/10.15171/ijhpm.2017.47

Berlant, L. (2007). Slow Death (Sovereignty, Obesity, Lateral Agency). Chicago Journals: Critical Inquiry, 33(4), 754-780. https://doi.org/10.1086/521568

Borruel, M. A. (2010). Estudio de carga de enfermedades. Argentina. Ministerio de Salud de la República Argentina. 
Burdiles Pinto, P. y Ortiz Pommier, A. (2021). El triaje en pandemia: fundamentos éticos para la asignación de recursos de soporte vital avanzado en escenarios de escasez. Revista Médica Clínica Las Condes, 32(1), 61-74. https://doi.org/10.1016/j.rmclc.2020.12.004

Butler, J. (1998). Actos performativos y constitución del género: un ensayo sobre fenomenología y teoría feminista. Debate feminista, 18, 296-314. https://doi.org/10.22201/cieg.2594066xe.1998.18.526

Butler, J. (2007). El género en disputa. Paidós.

Campbell, F. K. (2001). Inciting Legal Fictions: 'Disability's' date with Ontology and the Ableist Body of Law. Griffith Law Review, 10(1), 42-62. http://hdl.handle.net/10072/3714

Campbell, F. K. (2008). Refusing Able(ness): A Preliminary Conversation about Ableism. M/C Journal, 11(3). https://doi.org/10.5204/mcj.46

Campbell, S. M. y Stramondo, J. A. (2017). The Complicated Relationship of Disability and Well-Being. Kennedy Institute of Ethics Journal, 27, 151-184. https://doi.org/10.1353/ken.2017.0014

Canguilhem, G. (1971). Lo normal y lo patológico. Siglo XXI.

Coca Vila, I. (2021). Triaje y colisión de deberes jurídico-penal. Una crítica al giro utilitarista. InDret Penal. Revista para el análisis del Derecho, 1, 166-202. https://doi.org/10.31009/InDret.2021.i1.06

Disability Rights Washington (2020). RE: Complaint of Disability Rights Washington, Self Advocates in Leadership, The Arc of the United States, and Ivanova Smith Against the Washington State Department of Health (WA $\mathrm{DOH}$ ), the Northwest Healthcare Response Network (NHRN) and the University of Washington Medical Center (UWMC) [Carta en línea]. https://bit.ly/3eNpNZU

Dworkin, R. (2010). Keynote address. Justice for hedgehogs. Boston University Law Review, 90, 469-477. https://bit.ly/3wuaRqc

Emanuel, E. J., Persad, G., Upshur, R., Thome, B., Parker, M., Glickman, A., Zhang, C., Boyle, C., Smith, M., Phillips, J. P. (2020). Fair Allocation of Scarce Medical Resources in the Time of Covid-19. The New England Journal of Medicine, 382(21), 2049-2055. https://doi.org/10.1056/NEJMsb2005114

Extremo Ruano, A. G. (2016). Las fronteras de la justicia: de Rawls a Sen. Un análisis de la teoría de las capacidades. Facultad de Filosofía y Letras. Universidad de Valladolid. https://bit.ly/3hpeSaw

Fanon, F. (2001). L'an V de la révolution algérienne. La Découverte.

Fanon, F. (2009). Piel negra, máscaras blancas. Akal.

Fausto-Sterling, A. (2006). Cuerpos sexuados. La política de género y la construcción de la sexualidad. Melusina.

Fernández Romero, F. (2018). El activismo de las personas con discapacidad en torno al espacio urbano en Argentina. X Jornadas de Sociología de la Universidad Nacional de la Plata. https://bit.ly/3hmQrdS

Foucault, M. (2001). Los anormales. Fondo de Cultura Económica.

Foucault, M. (2008). El nacimiento de la clínica. Siglo XXI.

Fricker, M. (2007). Epistemic Injustice: Power and the Ethics of Knowing. Oxford University Press.

Fritsch, K. (2016). Cripping Neoliberal Futurity: Marking the Elsewhere and Elsewhen of Desiring Otherwise. Feral Feminisms, 5, 11-26. https://bit.ly/3tM8fSf

Gilabert, P. (2015a). The Socialist Principle "From Each According To Their Abilities, To Each According To Their Needs". Journal of Social Philosophy, 46, 197-225. https://doi.org/10.1111/josp.12096

Gilabert, P. (2015b). Human Rights, Human Dignity, and Power. En R. Cruft, M. Liao, y M. Renzo (Eds.) Philosophical Foundations of Human Rights. (pp. 196-213). Oxford University Press. https://doi. org/10.1093/acprof:0so/9780199688623.003.0011

Holzer, F. (2020). Bridging Exploitation and Structural Injustice. Revista Latinoamericana de Filosofía Política. Centro de investigaciones filosóficas, 9(3), 42-67. https://bit.ly/3w6Lha6

Kafer, A. (2013). Feminist, Queer, Crip. Indiana University Press.

Lee, J. Y., Ock, M., Jo, M., Son, W., Lee, H., Kim, S., Kim, H. J. y Lee, J. L. (2017). Estimating utility weights and quality-adjusted life year loss for colorectal cancer-related health states in Korea. Scientific Reports, 7, 1-9. https://doi.org/10.1038/s41598-017-06004-6

Maldonado Ramírez, J. (2020). Sentir la discapacidad en tiempos neoliberales: optimismo cruel y fracaso. Revista Nómadas, 52, 44-59. https://doi.org/10.30578/nomadas.n52a3 
Mathers C. D., Lopez A. D., y Murray C. J. L. (2006). The Burden of Disease and Mortality by Condition: Data, Methods, and Results for 2001. En A. D. Lopez, C. D. Mathers, M. Ezzati, D. T. Jamison, y C. J. L. Murray (Eds.) Global Burden of Disease and Risk Factors. (pp. 45-93). The International Bank for Reconstruction and Development / The World Bank.

McRuer, R. (2006). Crip Theory. Cultural Signs of Queerness and Disability. New York University Press.

McRuer, R. (2020). Capacidad corporal obligatoria y existencia discapacitada queer. Papeles del CEIC, 2, 1-12. https://doi.org/10.1387/pceic.21903

Millum, J. (2019). Putting a Number on the Harm of Death. En E. Gamlund, y C. Tollef Solberg (Eds.) Saving People from the Harm of Death. (pp. 61-75). Oxford University Press.

Ministerio de Sanidad, Servicios Sociales e Igualdad (2014) Encuesta Nacional de Salud. España 2011/12. Calidad de vida relacionada con la salud en adultos: EQ-5D-5L. Serie Informes monográficos $n^{\circ} 3$. MSSSI de España. https://bit.ly/3cHEDjb

Mogensen, A. (2019). Justifying and modeling acquisition of life potential for DALYs. En E. Gamlund, y C. Tollef Solberg (Eds.) Saving People from the Harm of Death. (pp. 48-60). Oxford University Press.

Moya Santander, L. (2018). Repolitizar lo cotidiano: cuerpos diverso-funcionales en los transfeminismos. Blog Laboratorio de Aragón [Gobierno] Abierto. https://bit.ly/3uT7lVy

Murray, C. J. (1994). Quantifying the burden of disease: the technical basis for disability-adjusted life years. Bulletin of the World Health Organization, 72(3), 429-445. https://bit.ly/3vjGvoP

Organización de Naciones Unidas. (2019). A/HRC/43/41. Derechos de las personas con discapacidad. Informe de la Relatora Especial sobre los derechos de las personas con discapacidad. Promoción y protección de todos los derechos humanos, civiles, políticos, económicos, sociales y culturales, incluido el derecho al desarrollo. Consejo de Derechos Humanos. 43er período de sesiones. https://bit.ly/3bm1CQf

Organización de Naciones Unidas. (2020). Informe de los Objetivos de Desarrollo Sostenible. División estadística de las Naciones Unidas. https://bit.ly/3hpGZGx

Organización de Naciones Unidas. (2021). Una recuperación económica a dos velocidades traerá más desigualdad, alerta el Fondo Monetario Internacional. Noticias ONU. https://bit.ly/3elRJxY

Organización Panamericana de la Salud. (2020). Evaluación de la Política de Equidad en la Salud en la Región de las Américas. Ediciones OPS.

Ortiz Amezquita, E. A. y Plata Castillo, L. (2011). Análisis de la utilidad de los años de vida ajustados a calidad en la toma de decisiones costo-efectivas. Revista CES Salud Pública, 2(2), 218-226. https://doi. org/10.21615/2001

Palacios, A. y Romañach, J. (2008). El Modelo de la Diversidad: una nueva visión de la bioética desde la perspectiva de las personas con diversidad funcional (discapacidad). Intersticios: Revista sociológica de pensamiento crítico, 2(2), 37-47. https://bit.ly/3og1gje

Pérez, M. (2020). La crisis de lo corriente y lo corriente como crisis. Blog Caja Negra Editora. https://bit. ly/3eLQnmj

Radi, B. (2019) Políticas trans y acciones afirmativas en los ámbitos universitarios. Conversaciones necesarias para deshacer el cisexismo. Aletheia, 10(19), e-026. https://doi.org/10.24215/18533701e026

Redacción LAVOZ (2021). Webinar abierto sobre los trabajadores de la economía informal en la crisis del Covid-19. La Voz, portal de noticias. https://bit.ly/3fdlPsy

Rivera López, E., Abal, F., Rekers, R., Holzer, F., Melamed, I., Salmún, D., Belli, L., Terlizzi, S., Alegre, M., Bianchini, A. y Mastroleo, I. (2020). Propuesta para la elaboración de un protocolo de triaje en el contexto de la pandemia de COVID-19. Revista de Bioética y Derecho, 50, 37-61. https://doi.org/10.1344/ rbd2020.50.31816

Savin, K., y Guidry-Grimes, L. (2020). Confronting Disability Discrimination During the Pandemic. The Hastings Center. https://bit.ly/3fjzBd3

Saxena, A., Bouvier P. A., Shamsi-Gooshki E., Köhler, J., y Schwartz, L. J. (2021). WHO guidance on ethics in outbreaks and the COVID-19 pandemic: a critical appraisal. Journal of Medical Ethics, 2021. https://doi. org/10.1136/medethics-2020-106959

Scientific Advisory Group for Emergencies (2020) DHSC/ONS/GAD/HO: Direct and indirect impacts of COVID-19 on excess deaths and morbidity, 15 July 2020. SAGE Collection. https://bit.ly/3bniG8f 
Shakespeare, T. (2006). Disability Rights and Wrongs. Routledge.

Smith, J. W. (2020). Coronavirus: Triage if Necessary, Health-Care Rationing, Never. The American Spectator. https://bit.ly/3eM1Ge3

Smith-Spark, L., y Gan, N. (2021). El mundo está en el peor momento de la crisis por covid-19. No tenía que ser así. CNN Español, portal de noticias. https://cnn.it/2RVqYgJ

Sociedad Española de Medicina Intensiva, Crítica y Unidades Coronarias. (2020). Recomendaciones éticas para la toma de decisiones en la situación excepcional de crisis por pandemia COVID-19 en las unidades de cuidados intensivos. (SEMICYUC). SEMICYUC Prensa. https://bit.ly/3w4fKoZ

Sociedad Española de Reumatología. (2015). EQ-5D. Cuestionario de Salud. Versión en español para US. Fundación SER. https://bit.ly/33Fy6Rj

Stramondo, J. (2020). Disability and the Damaging Master Narrative of an Open Future. Hastings Center Report: ¿For "All of Us"? On the Weight of Genomic Knowledge, 50(3), S30-S36. https://doi.org/10.1002/ hast.1153

Stramondo, J. A., y Campbell, S. M. (2020). Causing Disability, Causing Non-Disability: What's the Moral Difference? En A. Cureton, y D. Wasserman (Eds.) The Oxford Handbook of Philosophy and Disability. (pp. 1-27). Oxford University Press. https://doi.org/10.1093/oxfordhb/9780190622879.013.15

Thierer, J. (2019). ¿Qué son los estudios de Economía de la Salud? Parte 1. Comunicaciones. Sociedad Argentina de Cardiología. https://bit.ly/3bq6W5g

Valim, R. (2018). Estado de excepción. La forma jurídica del neoliberalismo. Derechos En Acción, 7(7). https:// doi.org/10.24215/25251678e167

Valverde Gefaell, C. (2015). De la necropolítica liberal a la empatía radical. Violencia discreta, cuerpos excluidos y repolitización. Icaria.

VOX Media. (2021). Coronavirus, explained. VOX Media, portal de noticias. https://bit.ly/3ylEjzY

Washington State Department of Health and the Northwest Healthcare Response Network. (2020). Scarce resource management \& crisis standards of care, Apr 10. Washington State Department of Health Website. https://bit.ly/2Rm62j7

Wasserman, D., Asch, A., Blustein, J., y Putnam, D. (2016). Disability: Health, Well-Being, and Personal Relationships. En E. N. Zalta (Ed.) The Stanford Encyclopedia of Philosophy. https://stanford.io/3bqQ3HD

White, D. B., y Lo, B. (2020). A Framework for Rationing Ventilators and Critical Care Beds During the COVID-19 Pandemic. JAMA, 323(18), 1773-1774. https://doi.org/10.1001/jama.2020.5046

Wieseler, C. (2020). Epistemic Oppression and Ableism in Bioethics. Hypatia, 35(4), 714-732. https://doi. org/10.1017/hyp.2020.38

Wilkinson, D. (2020). ICU triage in an impending crisis: uncertainty, pre-emption and preparation. Journal of Medical Ethics, 46, 287-288. https://doi.org/10.1136/medethics-2020-106226

Wolbring, G. (2008). The Politics of Ableism. Development, 51, 252-258. https://doi.org/10.1057/dev.2008.17

World Health Organization. (2008). Global Burden of Disease 2004 Update. World Health Organization Press.

Yarza de los Ríos, A., Angelino, A., Ferrante, C., Almeida, M. E., y Míguez Passada, M. N. (2019). Ideología de la normalidad: un concepto clave para comprender la discapacidad desde América Latina. En A. Yarza de los Ríos, L. Mercedes Sosa, y B. Pérez Ramírez (coords.) Estudios críticos en discapacidad: una polifonía desde América Latina. Consejo Latinoamericano de Ciencias Sociales (CLACSO).

\section{AUTOR}

Lautaro Leani. Profesor de Enseñanza Media y Superior en Filosofía por la Universidad de Buenos Aires. Integrante del Grupo de Investigación en Filosofía Aplicada y Políticas Queer (UBA/FILOCyT/SADAF). Fue Becario Estímulo UBACyT por la Facultad de Filosofía y Letras de la Universidad de Buenos Aires.

\section{Conflicto de intereses}

El autor declara que no existe conflicto de interés posible.

Financiamiento

No existió asistencia financiera de partes externas al presente artículo.

Agradecimientos

Este trabajo no hubiese sido posible sin los comentarios, sugerencias y correcciones de Moira Pérez e Ignacio Mastroleo. Asimismo, agradezco profundamente los intercambios enriquecedores generados en el Grupo de Investigación en Filosofía Aplicada y Políticas Queer. 